\title{
Experience with a Special Outpatient Antimicrobial Program at McMaster University Medical Centre in 1997
}

Ross A Pennie MD FRCPC, Verne Glavin MD FRCPC, Fiona M Smaill MB ChB FRCPC

$\mathrm{T}$ he administration of parenteral antibiotics to selected patients in their home or in outpatient facilities instead of in hospital has been perceived as having many benefits including reduced length of hospital stay $(1,2)$. The Infectious Disease and Microbiology Service at McMaster University Medical Centre, Hamilton, Ontario organizes every course of outpatient parenteral antimicrobial therapy among the hospital's population of inpatients. The program is called the Special Outpatient Antimicrobial Program (SOAP). During the study period, it was hospital policy that all hospitalized patients potentially suitable for home parenteral antibiotic therapy be referred for assessment by the Infectious Diseases and Microbiology Service (ID service). Some patients enrolled in the SOAP study had been referred to the ID service as outpatients and were enrolled in SOAP without initial hospitalization. Such outpatient referrals were not systematic, being at the discretion of local family doctors and emergency room physicians. Ambulatory patients who were given repeated injections of antibiotics solely under the supervision of the emergency department physicians without referral to the ID service were not included in this study.

The objectives of the SOAP pilot study were to determine the number of days patients spend on SOAP; the potential number of hospital days saved by early discharge on SOAP, the rate of complications with intravenous cannulae, the rate of adverse drug reactions, and the rate of therapeutic success and failure.

\section{PATIENTS AND METHODS}

All patients included in the study were assessed by the physicians of the ID service of McMaster University Medical Centre and were determined to require continued parenteral anti-infective therapy. Hospital inpatients were required to meet the following criteria at time of their discharge and enrolment in SOAP: afebrile for more than $24 \mathrm{~h}$, diminution in clinical signs of infection; minimal nursing care required, telephone in the home and follow-up appointment arranged with ID service (3). Outpatients could be enrolled in SOAP if they did not have life-threatening infections, required minimal nursing care, had a telephone in the home and agreed to careful medical follow-up.

A data collection form was completed prospectively for each SOAP patient by the infectious disease specialist responsible for the case. The following data were collected: demographic characteristics; nature of infection; anti-infective agents administered; number of days spent on SOAP; nature and number of intravenous devices inserted; complications of intravenous devices; adverse drug reactions; and clinical outcome. Clinical outcomes were categorized as cure (SOAP treatment was completed as planned and the infection resolved), cure requiring change in antibiotic therapy during SOAP, readmission while on SOAP or failure of infection to resolve.

The number of hospital days saved for each patient was calculated by counting the number of days the patient was on the SOAP (outpatient and parenteral) phase of their treatment.

Department of Pathology and Pediatrics, McMaster University Medical Centre, Hamilton, Ontario

Correspondence: Dr Ross Pennie, Pathology and Pediatrics, McMaster University Medical Centre, Room 3N27G, 1200 Main Street, Hamilton, Ontario L8S 4J9. Telephone 905-521-2100, fax 905-521-1703, e-mail rpennie@mcmaster.ca 
For patients continuing on oral antibiotics after completing the parenteral phase of their therapy, the time on oral therapy was neither included in the length of SOAP nor in the calculation of number of hospital days saved.

\section{RESULTS}

During the five-month observation period from June 1 to October 31, 1997, 44 patients were enrolled into the SOAP program. Of 44 patients, 25 (57\%) were enrolled upon hospital discharge and 19 were enrolled without being admitted. There were 37 adults, and seven children under age 16 years. The three most common diagnoses were cellulitis (10 patients), osteomyelitis (eight patients) and septic arthritis (five patients). The most commonly used antimicrobials were ceftriaxone (Rocephin, Hoffmann La Roche Limited) in 16 of 44 patients (36\%) (of whom four received all doses intramuscularly) and cefazolin in 12 of 44 patients (27\%). The duration of SOAP (days on parenteral antimicrobial therapy without being in hospital overnight) ranged from one to 90 days, with a median of 12 days and a mode of three days.

The estimated number of hospital days saved per patient ranged from 1 to 90 days. The median was 12 days saved, the mean 14.7 days, and the mode three days. There were 19 admissions avoided completely because patients began SOAP in the emergency department or the outpatient clinic. During the five-month (153 days) observation period, the total number of hospital days saved was 661 .

The clinical outcomes of the 44 SOAP patients were as follows: 36 (82\%) were cured without changes in antibiotic therapy, seven $(16 \%)$ were cured but required changes in antimicrobial therapy, one ( $2 \%$ ) required readmission, and none had infections that failed to resolve on SOAP.

Thirty-eight patients had intravenous devices for the delivery of their anti-infective therapy. The most commonly used intravenous device was a short peripheral vein cannula, used in 18 of 38 (47\%) patients with devices. There were nine of 38 patients $(24 \%)$ with flexible midline catheters inserted at the antecubital fossa, and six of 38 patients (16\%) with peripherally inserted central venous catheters. Five patients (13\%) had tunnelled subclavian catheters, two of which were inserted only for the SOAP therapy. Four of the 44 SOAP patients (9\%) received intramuscular ceftriaxone without ever having an intravenous line, and two young adults with acute osteomyelitis were discharged home on oral, not parenteral, antibiotics. Of the 38 patients who had intravenous devices, 28 (74\%) had only one device inserted during the entire outpatient portion of their antimicrobial therapy. Six patients (16\%) required two insertions of intravenous devices, two patients $(5 \%)$ required three insertions, two patients $(5 \%)$ required four insertions and two patients (5\%) required five insertions. There were no life-threatening complications with the devices, but one midline catheter became blocked (it was successfully opened with urokinase [Abbokinase, Abbott Laboratories Limited, Saint Laurent, Québec]) and one peripherally inserted central venous catheter leaked and had to be reinserted. There were no recognized line infections.

of the 44 patients, 38 (86\%) experienced no adverse drug reactions. Nonurticarial red rash occurred in two patients (4.5\%), hives occurred in two patients $(4.5 \%)$, severe diarrhea occurred in one patient $(2 \%)$ and severe nausea occurred in one patient $(2 \%)$.

\section{CONCLUSIONS}

Outpatient parenteral antibiotic therapy is a form of treatment associated with a high rate of successful outcome, with a minimum of problems with intravenous devices, few reinsertions of intravenous devices and few adverse drug reactions. This form of therapy has the potential for significant reductions in the length of hospital stay, particularly for infections of the soft tissues, bones and joints. The challenge will be to demonstrate to administrators that the reduced length of hospital stay translates into improved patient care and decreased cost per case.

\section{REFERENCES}

1. Tice AD. Experience with a physician-directed, clinic-based program for outpatient parenteral antibiotic therapy in the USA. Eur J Clin Microbiol Infect Dis 1995;14:655-61.

2. Tice AD. Alternate site infuson: The physician-directed, office-based model. J Intraven Nurs 1996;19:188-93.

3. Infectious Disease Society of America Practice Guidelines Committee. Practical guidelines for community-based parenteral anti-infective therapy. Clin Infect Dis 1997;25:787-801. 


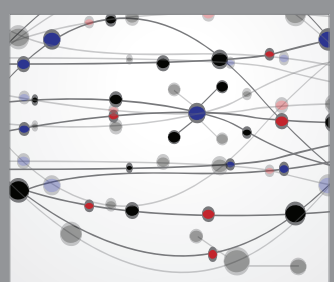

The Scientific World Journal
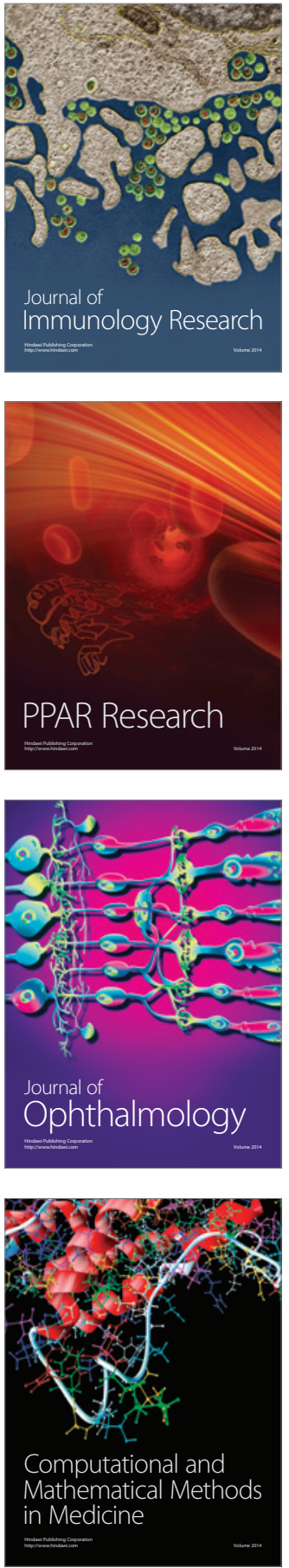

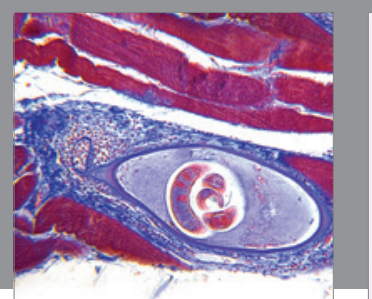

Gastroenterology Research and Practice

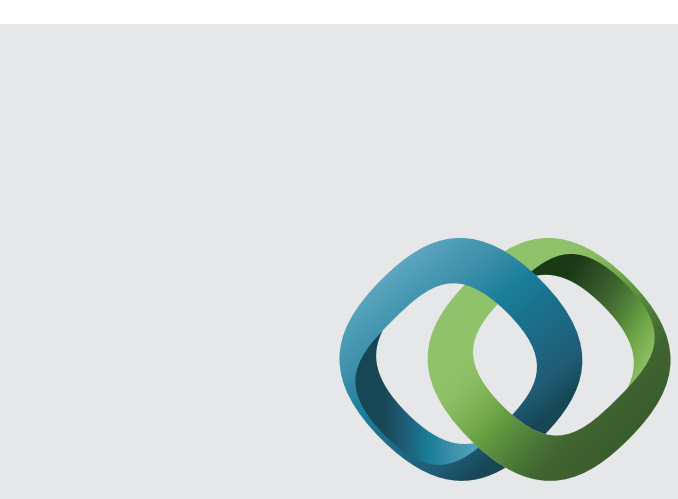

\section{Hindawi}

Submit your manuscripts at

http://www.hindawi.com
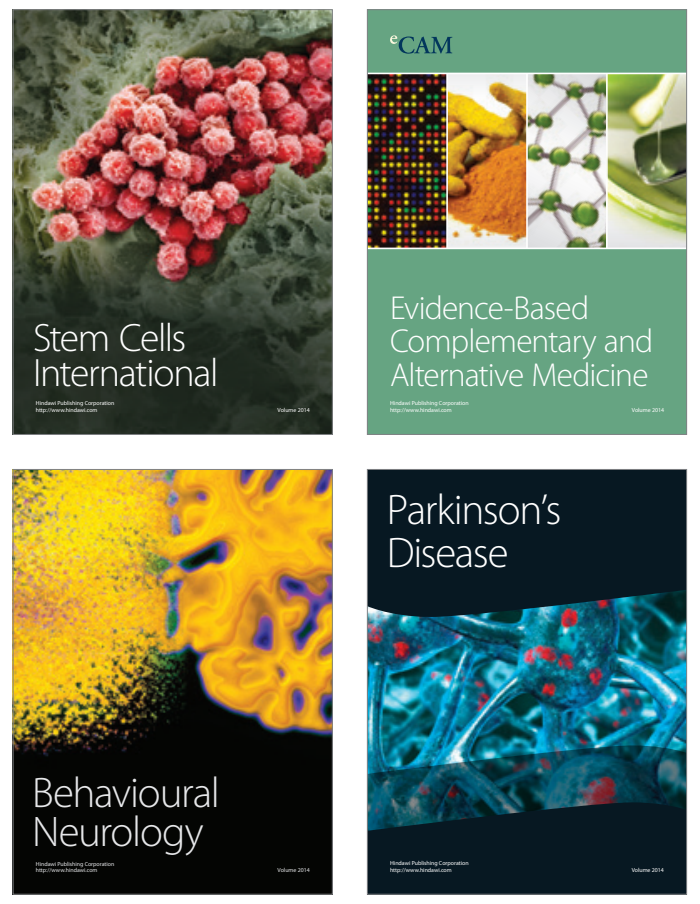
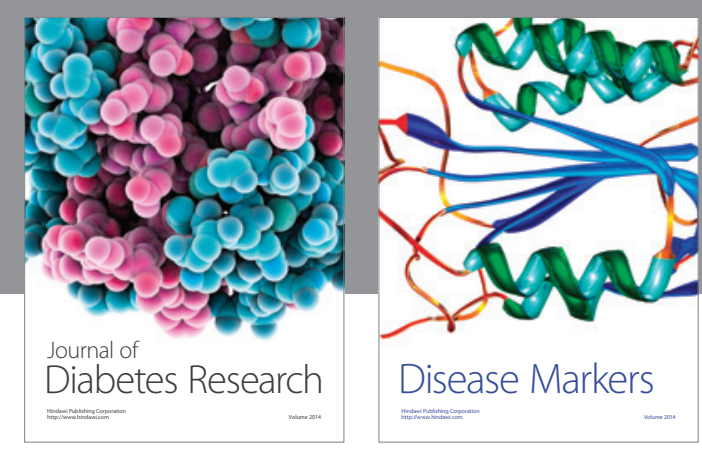

Disease Markers
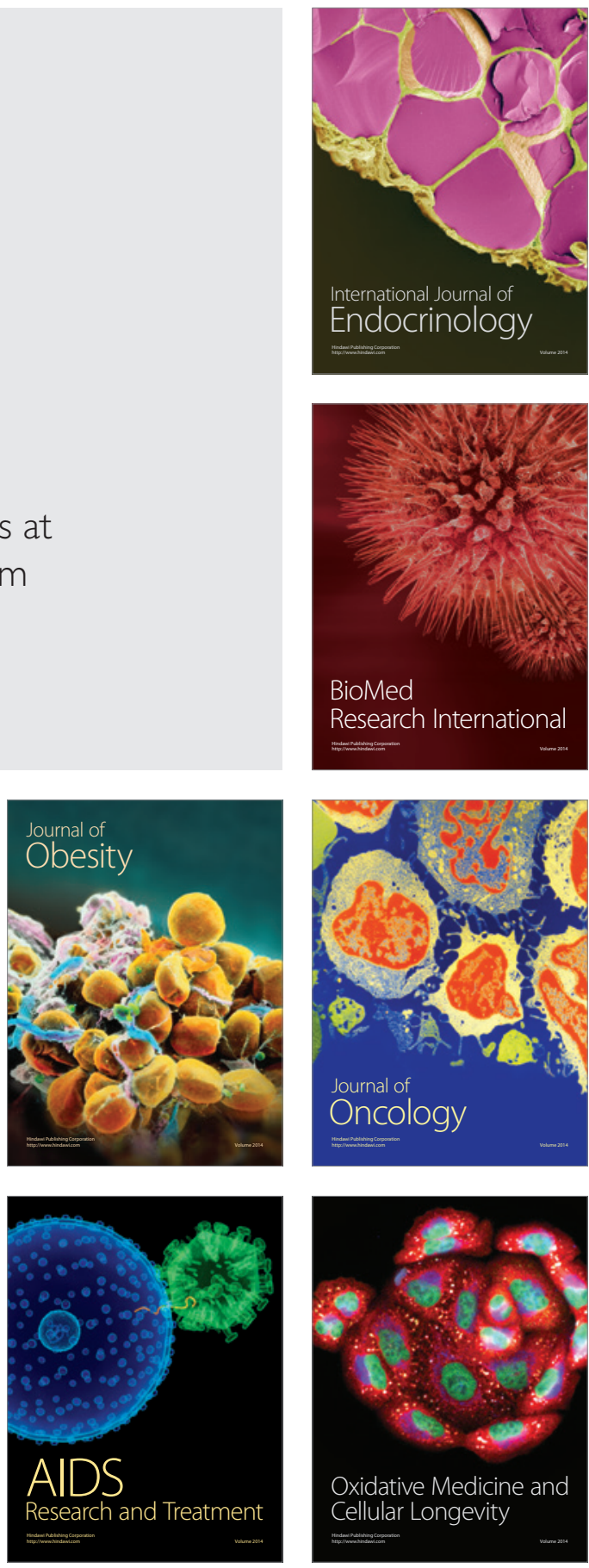